\title{
Posteriores kortikales Atrophie-Plus-Syndrom. Eine Kasuistik
}

\section{Posterior cortical atrophy-plus syndrome. A case report}

\author{
Autoren \\ Institute \\ 1 Gerontopsychiatrie, SHG-Kliniken Sonnenberg \\ 2 Klinik für Psychiatrie, Psychotherapie und Psychosomatik, \\ SHG-Kliniken Sonnenberg
}

Christian Saleh ${ }^{1}$, Ulrich Seidl ${ }^{2}$, Katharina Stegentritt ${ }^{1}$, Florian Schumacher ${ }^{1}$, Rose A. Fehrenbach ${ }^{1}$

Schlüsselwörter

Benson-Syndrom, Posteriore kortikale Atrophie,

Sehstörungen

Keywords

Benson Syndrome, Posterior cortical Atrophy, Visual

disturbances

eingereicht $\quad 11.09 .2019$

akzeptiert $\quad 18.03 .2020$

Bibliografie

DOI https://doi.org/10.1055/a-1149-2057

Fortschr Neurol Psychiatr 2020; 88: 528-531

(c) Georg Thieme Verlag KG Stuttgart · New York

ISSN 0720-4299

Korrespondenzadresse

Dr. Christian Saleh (Facharzt für Neurologie)

Gerontopsychiatrie

SHG-Kliniken Sonnenberg

Sonnenbergstraße 10

66119 Saarbrücken

Germany

Tel.: +49 6818890

Fax: +49 (0) 681/8 89-2197

E-Mail: chs12us75010@yahoo.com

\section{ZUSAMMENFASSUNG}

Die posteriore kortikale Atrophie (PCA) ist eine seltene neurodegenerative Erkrankung, die mit komplexen Sehstörungen einhergeht. Sie kann isoliert („PCA-pure“) oder zusammen mit anderen neurodegenerativen Störungen („PCA-plus“) auftreten. Die Diagnosestellung erfolgt meistens verzögert, da die Erkrankung wenig bekannt ist und oftmals initial eine ophthalmologische Ursache vermutet wird.

\section{ABSTRACT}

Posterior cortical atrophy (PCA) is a rare neurodegenerative disease, which manifests with complex visual disturbances. PCA can present in isolation ('PCA-pure') or in association with other neurodegenerative disorders ('PCA-plus'). Diagnosis is nevertheless frequently delayed, as PCA is a less known disease entity and initially a primary ocular disease is taken into consideration.

\section{Begriffserläuterung:}

- Optische Ataxie: Eine Sonderform der Ataxie. Eingeschränkte Fähigkeit, nach einem Objekt zu greifen, obwohl das Objekt gut gesehen wird.

- Agraphie: Verlust der Schreibfähigkeit. Unfähigkeit, Wörter zu schreiben, bei intaktem Intellekt und bei intakter Motorik.

- Akalkulie: Erworbene Schwäche im Umgang mit Zahlen trotz intaktem Intellekt.

- Okulomotorische (oder okuläre) Apraxie: Unfähigkeit zu willentlich schnellen horizontalen Augenbewegungen bei intakter Okulomotorik mit ausgleichenden Kopfbewegungen zur Blicksteuerung.

- Simultanagnosie: Unfähigkeit, mehr als ein Objekt auf einmal (simultan) zu erfassen, obwohl jedes Objekt getrennt richtig erkannt wird.

- Finger-Agnosie: Gestörte Fähigkeit im Benennen und Identifizieren der Finger der eigenen Hand oder die einer anderen Person.

- Apraxie: Neuropsychologische Störung in Ausführung zielgerichteter und geordneter Bewegung bei intakter Motorik. 
- Wernicke-Aphasie (sensorische Aphasie): Sprachstörung. Unfähigkeit, gesprochene oder geschriebene Sprache zu verstehen.

\section{Einführung}

Die posteriore kortikale Atrophie (PCA), auch als Benson-Syndrom bezeichnet, ist eine seltene progressive neurodegenerative Erkrankung, die 1988 von D. Frank Benson erstmals beschrieben wurde [1].

Die Erkrankung manifestiert sich typischerweise in der späten fünften bis frühen sechsten Lebensdekade. Die Prävalenz ist nicht bekannt. Benson und Kollegen berichteten von 5 Patienten mit progressiver Demenz, frühem Auftreten komplexer visueller Störungen einschließlich Bálint-Syndrom (optische Ataxie, Simultanagnosie und okuläre Apraxie), Gerstmann-Syndrom (Agraphie, Akalkulie, links-rechts-Verwechslung und Finger-Agnosie) sowie Zeichen einer Wernicke-Aphasie. Zu der klinischen Symptomatik passend findet sich bildmorphologisch eine parieto-okzipitale Atrophie. Während einige Autoren die PCA als eine „visuelle Variante" der Alzheimer-Erkrankung beschreiben [2], betonen andere, dass die PCA eine eigene neurodegenerative Erkrankung darstellt $[3,4]$. Die Unterscheidung zwischen PCA und Alzheimer-Erkrankung basierte auf Autopsiebefunden [4-6]; so stellten Tang-Wai und Kollegen in post-mortem-Untersuchungen bei Patienten mit PCA fest, dass neurofibrilläre Bündel in den okzipitalen BrodmannArealen 17 und 18 in signifikant höheren und im Hippocampus in signifikant niedrigeren Dichten präsent waren als bei Patienten mit „typischer“ Demenz vom Alzheimer-Typ [5]. Neuropathologische Befunde zeigen, dass Patienten mit PCA im temporalen und präfrontalen Kortex vergleichbar weniger Läsionen aufweisen als Patienten mit Demenz vom Alzheimer-Typ [3]. Jedoch bereitete die strikte Trennung zwischen PCA und anderen neurodegenerativen Erkrankungen Schwierigkeiten in der Zuordnung jener Patienten, die sowohl die Kriterien einer PCA erfüllten als auch Symptomatiken und/oder neuropathologische Befunde aufwiesen, die sich mit weiteren neurodegenerativen Erkrankungen überlappten. Auf dieser Grundlage wurde 2017 ein Consensus Statement formuliert, das die PCA-Diagnose erweiterte, um die Einheitlichkeit der PCA-Kriterien und der Diagnosestellung zu gewährleisten [7]. Nach diesem Consensus-Bericht wird zwischen einer reinen PCA-Form („PCA-pure“) und einer „PCA-plus“-Form unterschieden. Zeigt der Patient alleine klinische Symptome wie visuelle Störungen, eine Simultanagnosie, Elemente eines Gerstmann- oder eines Bálint-Syndroms und radiologisch eine parieto-okzipitale Atrophie, also eine „klassische“ PCA Symptomatik, so liegt demgemäß eine isolierte, d.h. eine „PCA-pure“-Form vor. Finden sich hingegen neben den Leitsymptomen eines isolierten PCA-Syndroms auch Symptome anderer neurodegenerativer Erkrankungen, z.B. visuelle Halluzinationen wie bei der Lewy-Körperchen-Demenz (LKB), wird eine „PCA-plus“ diagnostiziert [3, 4]. Die Diagnose kann beim Nachweis einschlägiger Biomarker präzisiert werden in $P C A-A D$ („Alzheimer Disease“), PCA-LBD („Lewy-Body Disease“), PCA-CBD („Cortico-basal degeneration“) und PCA-prion [3, 4]. Trotz der Erweiterung der diagnostischen Kriterien wird eine PCA meistens verzögert festgestellt, nicht zuletzt deshalb, da für die visuellen Störungen zunächst ophtalmologische Ursachen gesucht werden.
- In unserer Kasuistik beschreiben wir einen Patienten mit einem PCA-plus-Syndrom.

\section{Fallbeschreibung}

Ein 63-jähriger Patient wurde notfallmäßig bei initial delirantem Krankheitsbild aufgenommen. Anfänglich war der Patient in der Abteilung der Inneren Medizin wegen Exazerbation einer chronisch obstruktiven Lungenkrankheit behandelt worden. Fremdanamnestisch war zu erfahren, dass der Patient seit dem erst kurz zurückliegenden Tod der Ehefrau zunehmend verwirrt und häufig desorientiert gewesen sei. Der Patient habe im Vorfeld der Aufnahme im Rahmen eines deliranten Zustand halluziniert und sei nicht orientiert gewesen. Als der Patient sich nahezu unbekleidet auf die Straßenbahnschienen begeben habe, sei die notfallmäßige Verlegung in unsere Klinik erfolgt. An Vorerkrankungen waren Asthma, eine Pankreatitis, Diabetes mellitus Typ 2 ohne Komplikationen sowie eine gastrojejunale Anastomose bekannt. In den ersten Tagen des stationären Aufenthaltes fielen sowohl visuell-räumliche Orientierungsstörungen als auch visuo-konstruktive Störungen auf, welche zu deutlichen Einschränkungen im Alltag führten. Anamnestisch war zu erfahren, dass der Patient seine Kinder zuletzt nicht mehr erkennen konnte. In der ersten testpsychologischen Untersuchung war der Patient in der Lage, biographische Angaben inhaltlich und chronologisch gut abzurufen. Im Kurztest zur Erfassung von Gedächtnisund Aufmerksamkeitsstörungen (SKT) konnte er Zahlen auf Klötzen zwar problemlos vorlesen, war jedoch nicht in der Lage, diese zu ergreifen und der Größe nach zu sortieren. Es zeigten sich Defizite im Altgedächtnis sowie Störungen der Merkfähigkeit und der Aufmerksamkeit. In der CERAD-Testbatterie (CERAD = Consortium to Establish a Registry for Alzheimer's Disease) war das Benennen schwer beeinträchtigt bei ansonsten intakter verbaler Flüssigkeit. Bei der Visuokonstruktion hatte der Patient, der sonst gut lesen konnte, deutliche Schwierigkeiten, die gesehenen Formen als solche zu erkennen bzw. zu verarbeiten. Im Uhrentest zeigte sich eine schwere visuell-räumliche Desorganisation mit einem Score von 5 ( $\triangleright$ Abb. 1). Im Rahmen der Testung fielen beim Patienten eine optische Ataxie und eine Apraxie auf. So griff er neben Zielobjekte und konnte gestellte Instruktionen nur schwer oder gar nicht umsetzen. Die kranielle Magnetresonanztomographie (cMRT) zeigte eine ausgeprägte beidseitige parieto-okzipital betonte Atrophie ( $\triangleright$ Abb. 2 a, b). Die Liquordiagnostik wies für eine Alzheimer-Demenz typische Befunde auf, mit einem Beta-Amyloid-Quotienten von 4\% (normal: > 5,5\%) und einer Phospho-Tau-Konzentration von $67,67 \mathrm{pg} / \mathrm{ml}$ (normal: < 66). Protein 14-3-3 wurde nicht nachgewiesen. Ferner zeigten sich eine grenzwertige Blut-LiquorSchranken-Störung, aber keine oligoklonalen Banden und keine Hinweise auf eine intrathekale IgG-Synthese. Die weitere laborchemische Diagnostik, einschließlich Vitamin B12, Folsäure und Treponema-Pallidum-Hämagglutinations-Assay, erbrachte keinen richtungsweisenden Befund. Die im Verlauf durchgeführte testpsychologische Untersuchung bestätigte mittelgradige Defizite im Neugedächtnis sowie schwere 


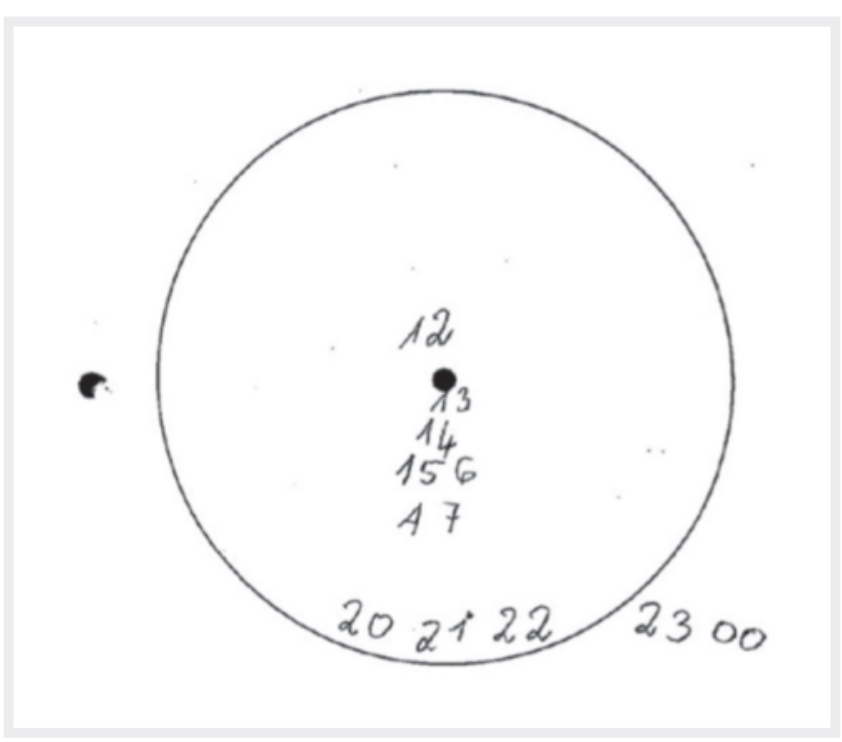

-Abb. 1 Uhrentest mit einer schweren räumlichen Desorganisation.

Störungen der Benennfunktion, der Visuokonstruktion und der visuell-räumlichen Organisation. Wir stellten, basierend auf dem klinischen Befund und der organischen Diagnostik, einschließlich des Befundes der bilateralen parieto-okzipitalen Atrophie in der MRT, die Diagnose einer PCA-plus, am ehesten vom Alzheimer-Typ.

\section{Diskussion}

Die PCA ist, wie erwähnt, charakterisiert durch eine Vielzahl von Symptomen, v.a. einer visuellen Agnosie (die Unfähigkeit, Objekte zu erkennen, ohne eine okuläre oder semantische Störung), einer Simultanagnosie, einhergehend mit einer progressiven Demenz [1, 6]. Bildmorphologisch kann die PCA eine rechtsbetonte parieto-okzipitale Atrophie zeigen, die mit der klinischen Symptomatik einer massiven räumlich-visuellen Störung korreliert ist. Singh et al. beschreiben, dass distinkte Veränderungen des zerebralen Metabolismus durch Positronenemissionstomographie aufgezeigt werden können [8]. Der veränderte fokale zerebrale Metabolismus bei der PCA ist nicht vollständig erklärt, aber sehr wahrscheinlich Ursache der neurodegenerativen Veränderungen, die zu einer morphologisch wie funktionellen Störung des neuronalen Netzwerkes führen [9]. Degenerativ neurologische Erkrankungen, wie z.B. die Kortikobasale Degeneration (CBD), die Lewy-Body-Demenz (LBD), die Multisystematrophie (MSA) oder die Supranukläre Blickparese (PSP), gehen wie die PCA mit fokal-hypometabolischen Alterationen einher, die im Rahmen der neurodegenerativen Alterationen der Grunderkrankung verstanden werden [10]. Patienten mit Simultanagnosie können einen Hypometabolismus im rechten Okzipitallappen aufweisen, hingegen kann die optische Ataxie mit einem Hypometabolismus im linken Okzipitallappen und die okulomotorische Apraxie mit einem Hypometabolismus im linken Parietallappen einher gehen [8]. Die „reine“ PCA-Form („PCA-pure“) muss differenzialdiagnostisch vor allem von einer Creutzfeld-Jakob-Erkrankung (CJK), Lewy-Körperchen-Demenz (LKD), einem Tumor oder einem Schlaganfall

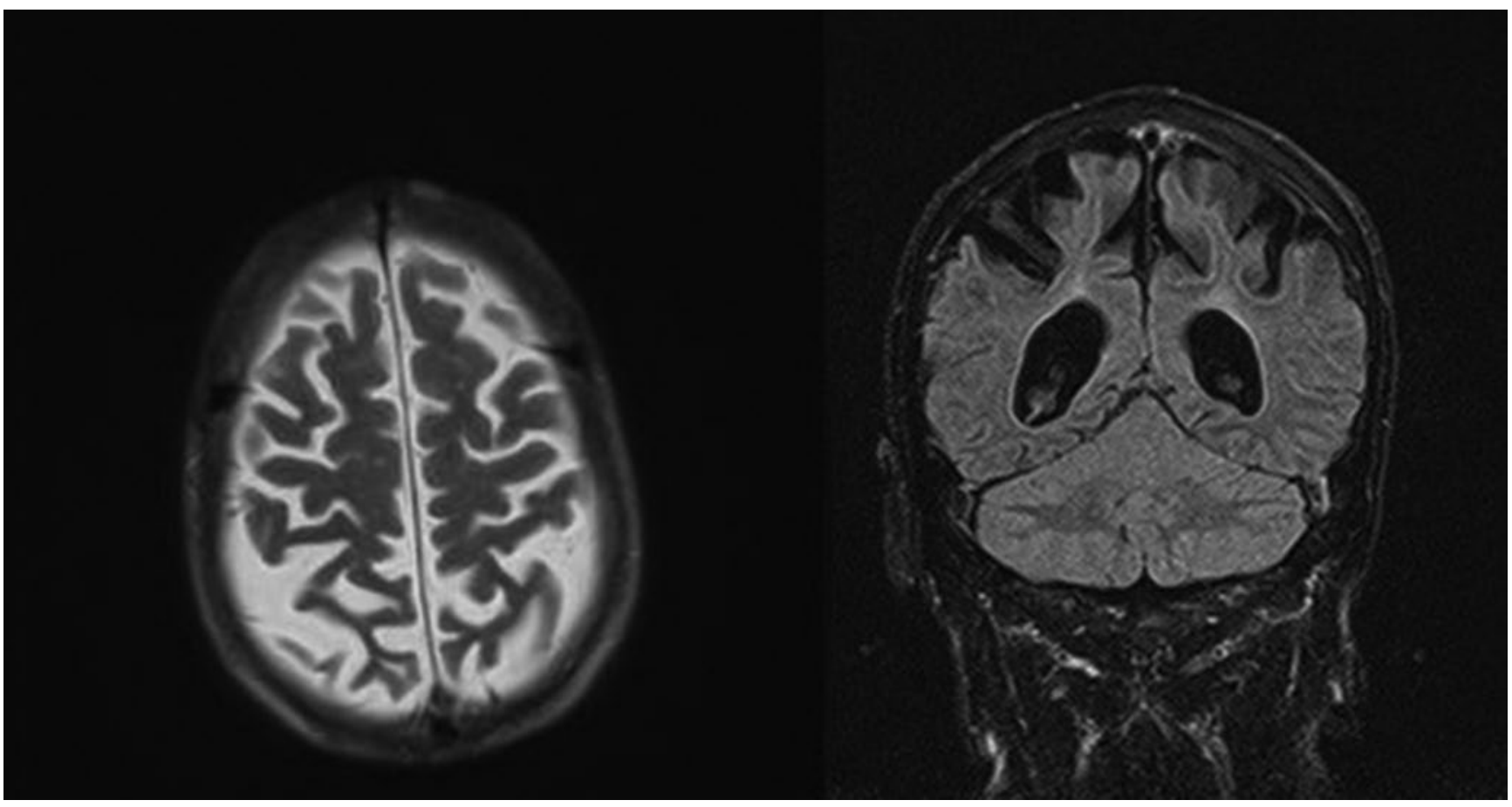

Abb. 2 a) Schädel-MRT in T2-Gewichtung, axiale Schnittführung, b) Schädel-MRT Flair-Sequenz, koronare Schnittführung. Zu sehen ist eine deutliche bilaterale parietale Atrophie. 
abgegrenzt werden [11, 12]. Bei der CJK ist der Verlauf schnell fortschreitend, mit charakteristischen periodischen Veränderungen im EEG sowie einem Nachweis des Proteins 14-3-3. Optische Halluzinationen, Antipsychotika-Unverträglichkeit, fluktuierende Vigilanz sowie Parkinsonismus sind hingegen charakteristisch für LKD.

Unser Patient zeigte zu den PCA-typischen-Symptomen von visuellen Störungen zudem klinisch wie laborchemisch eine Alzheimer-typische Konstellation, d.h. mnestische Störungen, die sich bereits in der Frühphase der Erkrankung manifestierten und nicht erst im späteren Verlauf. Auf der Grundlage dieser klinischlaborchemischen Konstellation - einerseits charakteristisch für $A D$ und andererseits für PCA - wurde die Diagnose eines PCAPlus-Syndroms/PCA-AD gestellt.

Aktuell besteht keine klare Empfehlung für die pharmakologische Therapie der PCA. Acetylcholinesterase-Inhibitoren, die in der Behandlung der Alzheimer-Erkrankung eingesetzt werden, können off-label verabreicht werden $[3,13]$. Kognitiv-mnestische Funktionen sind in der „reinen“ PCA-Form nur im fortgeschrittenen Verlauf beeinträchtigt. Der Patient mit einer „reinen“ PCAForm besitzt daher im Frühstadium noch Krankheitseinsicht, was zu einem hohen Leidensdruck und zu einer depressiven Symptomatik führen kann [13, 14]. Frühzeitig sollten Kompensationsstrategien erlernt und eine medikamentöse antidepressive Behandlung in Betracht gezogen werden.

\section{Konklusion}

Die PCA ist eine progressive neurodegenerative Erkrankung. Die Diagnose der PCA kann durch überlagernde Symptome erschwert, verzögert oder sogar versäumt werden. Charakteristisch für die PCA sind graduell manifestierende visuell-räumliche Defizite und mnestische Defizite, die sich sowohl in der Frühphase (PCA-AD) als auch in der Spätphase (eher bei der isolierten PCA-Form) manifestieren können. Daher sollte bei einer demenziellen Symptomatik, die mit derartigen Defiziten einhergeht, frühzeitig an eine PCA gedacht und diese gemäß den aktualisierten Empfehlungen differenzialdiagnostisch abgeklärt werden. Therapeutische Maßnahmen sind bis dato nur symptomatisch.
Interessenkonflikt

Die Autoren geben an, dass keine Interessenkonflikte vorliegen.

\section{Literatur}

[1] Benson DF, Davis RJ, Snyder BD. Posterior cortical atrophy. Arch Neurol 1988; 45: 789-793

[2] Pantel J, Schroder J. Posterior cortical atrophy - a new dementia syndrome or a form of Alzheimer's disease? Fortschr Neurol Psychiatr 1996; 64: 492-508

[3] Maia da Silva MN, Millington RS, Bridge $\mathrm{H}$ et al. Visual dysfunction in posterior cortical atrophy. Front Neurol 2017; 16(8): 389

[4] Mendez MF, Ghajarania M, Perryman KM. Posterior cortical atrophy: Clinical characteristics and differences compared to Alzheimer's disease. Dement Geriatr Cogn Disord 2002; 14: 33-40

[5] Victoroff J, Ross GW, Benson DF et al. Posterior cortical atrophy. Neuropathologic correlations. Arch Neurol 1994; 51: 269-274

[6] Tang-Wai DF, Graff-Radford NR, Boeve BF et al. Clinical, genetic, and neuropathologic characteristics of posterior cortical atrophy. Neurology 2004; 63: 1168-1174

[7] Crutch SJ, Schott JM, Rabinovici GD et al. Consensus classification of posterior cortical atrophy. Alzheimers Dement 2017; 13: 870-884

[8] Singh TD, Josephs KA, Machulda MM et al. Clinical, FDG and amyloid PET imaging in posterior cortical atrophy. J Neurol 2015; 262(6): 1483-1492

[9] Nestor PJ, Caine D, Fryer TD et al. The topography of metabolic deficits in posterior cortical atrophy (the visual variant of Alzheimer's disease) with FDG-PET. J Neurol Neurosurg Psychiatry 2003; 74(11): 1521-1529

[10] Thobois S, Prange S, Scheiber $C$ et al. What a neurologist should know about PET and SPECT functional imaging for parkinsonism: A practical perspective. Parkinsonism Relat Disord 2019; 59: 93-100

[11] Whitwell JL, Graff-Radford J, Singh TD et al. (18)F-FDG PET in posterior cortical atrophy and dementia with lewy bodies. J Nucl Med 2017; 58 (4): 632-638

[12] Croisile B Benson's syndrome or posterior cortical atrophy. Orphanet Encyclopedia. September 2004

[13] Wolf RC, Uttner I, Osterfeld ND. Depressive symptoms in a case of "posterior cortical atrophy”. Fortschr Neurol Psychiatr 2010; 78: 226-229

[14] Karner E, Jenner C, Donnemiller E et al. The clinical syndrome of posterior cortical atrophy. Nervenarzt 2006; 77: 208-214 